\title{
Intramodal and cross-modal matching of emotional expression in young children with autism spectrum disorders
}

\author{
Soichiro Matsuda ${ }^{\mathrm{a}, *}$, Junichi Yamamoto ${ }^{\mathrm{a}, \mathrm{b}}$ \\ ${ }^{a}$ Department of Psychology, Keio University, Tokyo, Japan \\ ${ }^{\mathrm{b}}$ CREST, Japan Science and Technology Agency, Saitama, Japan
}

\section{A R T I C L E I N F O}

\section{Article history:}

Received 1 October 2014

Accepted 13 November 2014

\section{Keywords:}

Affective prosody

Autism

Cross-modal matching

Emotion comprehension

Facial expression

\begin{abstract}
A B S T R A C T
Children with autism spectrum disorders (ASDs) exhibit difficulties in their comprehension of others' emotions. According to a variety of experimental procedures, many of them can be classified into two types according to the modality of their stimuli and responses. These are intramodal (visual stimulus-visual stimulus) and cross-modal (auditory stimulus-visual stimulus) matching. Previous studies tested both intramodal and crossmodal matching only in adolescents with ASD, although young children with ASD have also been found to have difficulties with cross-modal matching but not intramodal matching. The purpose of this study was to compare the intramodal and cross-modal matching of emotional expression in young children with ASD and typically developing (TD) children. Ten children with ASD aged 4-8 and 22 developmental age (DA)-matched TD children aged 3-6 participated in this study. Pictures of facial expressions were used as visual stimuli, and affective prosodies were used as auditory stimuli. The results showed that the children with ASD were less accurate than the TD children in cross-modal matching but equally accurate on intramodal matching. These findings are discussed along with the modality of stimuli and responses, and the ages of the participants.
\end{abstract}

(c) 2014 Elsevier Ltd. All rights reserved.

\section{Introduction}

Autism spectrum disorders (ASDs) are neurodevelopmental disorders characterized by distinct social deficits (American Psychiatric Association, 2013). Individuals with ASD have difficulties particularly in their comprehension of others' emotions conveyed from nonverbal cues, such as facial expressions or affective prosodies (e.g., Braverman, Fein, Lucci, \& Waterhouse, 1989; Hobson, 1986a, 1986b; Macdonald et al., 1989; Tantam, Monaghan, Nicholson, \& Stirling, 1989). However, the results of previous studies have yielded inconsistent findings. Some studies found that individuals with ASD face difficulties in understanding other's emotions (e.g., Bormann-Kischkel, Vilsmeier, \& Baude, 1995; Celani, Battacchi, \& Arcidiacono, 1999; Davies, Bishop, Manstead, \& Tantam, 1994). Other studies did not find such deficits (e.g., Capps, Yirmiya, \& Sigman, 1992; Gepner, Deruelle, \& Grynfeltt, 2001; Prior, Dahlstrom, \& Squires, 1990). Matsuda and Yamamoto (2013, 2014) suggested that these inconsistencies occurred due to variations in the experimental tasks and ages of the participants.

Abbreviations: ASD, autism spectrum disorders; TD, typically developing; CA, chronological age; DA, developmental age; KSPD, Kyoto Scale of Psychological Development 2001.

* Corresponding author at: Department of Psychology, Keio University, 2-15-45 Mita, Minato-ku, Tokyo, Japan. Tel.: +81 334534521 ;

fax: +8135427 1578 .

E-mail address: atom.opens.the.stargate@gmail.com (S. Matsuda). 
Previous emotion comprehension studies can be classified into two types according to the modality of their stimuli and responses. These are intramodal (i.e., visual stimulus-visual stimulus) and cross-modal (i.e., auditory stimulus-visual stimulus) matching. In some studies where intramodal matching was used, pictures or movies showing facial expressions were presented as sample stimuli, and either facial expressions or texts were used as response choices. In other studies where cross-modal matching was used, affective prosodies representing different emotional states were presented as sample stimuli, and illustrations, texts, or both were used as response choices.

To date, few studies have used both intramodal and cross-modal matching (Jones et al., 2011; Loveland et al., 1997). Jones et al. (2011) tested adolescents with ASD (mean age: 15 years, 6 months), and as did Loveland et al. (1997) (mean age: 12 years, 2 months). In both studies, the results showed no difference in intramodal and cross-modal matching between adolescents with ASD and typically developing (TD) adolescents. However, to our knowledge, no study has compared the intramodal and cross-modal matching of emotional expressions in younger children with ASD.

It is possible that the difficulties children with ASD experience in cross-modal matching of emotional expressions are related to their age. Studies of older children with ASD, aged 12-16 years, have found that they have no difficulty in crossmodal matching (Baker, Montgomery, \& Abramson, 2010; Grossman, Bemis, Plesa-Skwerer, \& Tager-Flusberg, 2010; Jones et al., 2011; Paul, Augustyn, Klin, \& Volkmer, 2005). Meanwhile, studies of younger children with ASD, aged 9-10 years, have reported significant group differences (Boucher, Lewis, \& Collis, 2000; Peppé, McCann, Gibbon, O’Hare, \& Rutherford, 2007). These results suggest that younger children with ASD might have difficulties in cross-modal matching of emotional expressions.

On the other hand, in intramodal matching of emotional expressions, children with ASD might have no difficulties regardless of their age. Previous studies on intramodal matching have reported that both younger (aged 6-11 years; Fink, de Rosnay, Wierda, Koot, \& Bageer, 2014; Grossman, Klin, Carter, \& Volkmar, 2000; Lacroix, Guidetti, Rogé, \& Reilly, 2009; Rosset et al., 2008) and older (aged 12-15 years; Castelli, 2005; Loveland et al., 1997) children with ASD, and TD children performed equally well. These results indicate that intramodal matching is not difficult for either young or old children with ASD.

The purpose of the present study was to compare intramodal and cross-modal matching of emotional expressions in young children with ASD and TD children. Ten boys with ASD (mean age: 6 years, 2 months) participated in this study. They were younger and had lower developmental ages (DAs) than the participants in previous studies. The intramodal matching required the children to match pictures of facial expressions across different facial identities. The cross-modal matching required them to match an affective prosody to the corresponding picture of the facial expression. We used four basic emotions, happy, surprised, angry, and sad, for both intramodal and cross-modal matching.

\section{Methods}

\subsection{Participants}

Ten boys with ASD participated in this study. The diagnosis for nine children was autistic disorder, and for one was PDDNOS. Their diagnoses were provided by an outside professional, according to the criteria of the DSM-IV-TR (American Psychiatric Association, 2000). The average chronological age (CA) of this group was 6.31 years (SD: 1.20, range 4.5-8.3 years), and the average DA was 5.48 years (SD: 1.12, range 3.4-6.8 years). The eight participants' DA was assessed by The Kyoto Scale of Psychological Development 2001 (KSPD; Ikuzawa, Matsushita, \& Nakase, 2002). The remaining two participants' DA was assessed by the Japanese version of the Stanford-Binet Intelligence Scale (Tanaka Institute for Educational Research, 2003). KSPD and the Japanese version of the Stanford-Binet Intelligence Scale have high correlation in young children with ASD (Koyama, Osada, Tsujii, \& Kurita, 2009).

There were 22 TD participants without a psychological diagnosis (16 males and 6 females). They were matched by their average age to the DA of the ASD group. The average CA was 5.47 years (SD: 0.77, range 3.8-6.9 years).

The difference between the mean CA of each group approached significance; children in the ASD group were older than children in the TD group $(t=2.40, p=0.023, r=0.40)$. There were no significant differences between the mean DA in the ASD group and CA in the TD group $(t=0.05, p=0.960, r=0.01)$.

\subsection{Stimuli}

\subsubsection{Visual stimuli (facial expressions)}

The visual stimuli comprised eight pictures of facial expressions. The pictures comprised two different faces representing each of the four emotions: happy, surprised, angry, and sad. The two faces were male and Japanese. These were six emotions from Ekman (1992). Based on the results by Ekman et al. (1987), fear and disgust emotions were excluded from this study because Japanese people had difficulty identifying them. The pictures were printed on $9.5 \mathrm{~cm} \times 13.0 \mathrm{~cm}$ white laminated cards.

\subsubsection{Auditory stimuli (affective prosodies)}

The auditory stimulus used was the word sensei ("teacher" in Japanese). It was spoken in a voice representing each of the four emotions. These words were spoken in vivo by a male in his twenties. 


\subsubsection{Rating}

Prior to the experiment, 11 typical adults rated the pictures of facial expressions and example recordings of the affective prosodies. These pilot participants were asked to rate them on a 7-point Likert scale for each emotion and on the Affect Grid (Russell, Weiss, \& Mendelsohn, 1989) for pleasure-displeasure and arousal-sleepiness. The results of these ratings are presented in the Appendix.

\subsection{Procedure}

Each session in this study comprised eight trials, and sample stimuli were presented in semi-random order. The positions of the comparison stimuli changed at the beginning of each session and were counterbalanced across sessions.

Before testing both intramodal and cross-modal matching, training trials were conducted. When the child achieved $100 \%$ correct responses in each training session, the test session started. There was one training session and two testing sessions.

\subsubsection{Intramodal matching (facial expression-facial expression)}

2.3.1.1. Training session. The training session were conducted to ensure that the children were able to discriminate the facial expressions. During the training session, only one model's facial expressions were used. The child was shown a picture of a facial expression and was then instructed to "choose the same" from among the response choices. The child was required to look at the sample picture of a facial expression, and either point to an identical picture of the facial expression shown among the response choices or to hand the picture to the experimenter. The experimenter did not provide feedback on correct or incorrect responses. When the child did not respond within approximately $5 \mathrm{~s}$ after the first presentation of the sample stimulus, the experimenter presented it again. Irrespective of whether the child responded or did not respond within approximately $5 \mathrm{~s}$ after the second presentation of the sample stimulus, the experimenter would immediately start the next trial. Correct responses were recorded when the child chose the identical picture of the facial expression presented. Incorrect responses were recorded when the child did not choose the corresponding picture or did not respond within approximately $5 \mathrm{~s}$ after the second presentation of the sample stimulus.

2.3.1.2. Test sessions. During the test session, two models' facial expressions were used. One was used as a sample stimulus and the other as a comparison stimulus. All the other procedures were the same as in the training session.

\subsubsection{Cross-modal matching (affective prosody-facial expression)}

The basic procedure for cross-modal matching was the same as that for intramodal matching, except for the stimuli that were used.

2.3.2.1. Training session. The training session was conducted to ensure that the children understood the auditory-visual matching. During the training session, the sample stimuli comprised four animal calls (dog, cat, pig, and chicken), and the response choices were picture cards corresponding to each of the animals. Each trial began with the experimenter's instruction, "Choose which animal is calling." The child then listens to the animal call (e.g., wan-wan, which is "bow-wow" in Japanese) presented by the experimenter's voice. Then, the child would either point to the corresponding picture of the animal or hand the picture to the experimenter.

2.3.2.2. Test sessions. During the test session, the sample stimulus was the word sensei ("teacher" in Japanese), with the prosody of one of four emotions. Pictures of facial expressions representing the four emotions were used as comparison stimuli. The trial began with the experimenter's instruction, "Choose which face is talking." The experimenter would then present a sample stimulus to the child, while hiding his face behind a black board. The child would listen to the sample of affective prosody, and either point to the picture with the corresponding facial expression or hand the picture to the experimenter.

\subsection{Statistical analysis}

The analyses were conducted using the Statistical Package for Social Sciences (SPSS/Win in 22.0). Due to the small sample sizes and small number of variables in the data, nonparametric tests were used to contrast the percentage of correct response (Wilcoxon signed-rank tests or Mann-Whitney $U$-tests). The $z$ value was used to calculate an effect size $r$ (Fritz, Moriis, \& Richler, 2012). All $p$ values reported are two-tailed.

\section{Results}

All children met the criteria to start the test sessions, based on the training session of intramodal and cross-modal matching. 


\subsection{Group differences for two matching tasks}

Fig. 1 shows a box plot comparison of intramodal and cross-modal matching for children with ASD and TD children.

\subsubsection{Intramodal matching}

In the intramodal matching, the mean percentage of correct responses was $85.0 \%(S D=29.0)$ for children with ASD and 98.9\% (SD = 3.1) for TD children. There was no difference between the two groups in terms of the percentage of correct responses (Mann-Whitney test: $Z=1.75, p=0.081, r=0.31$ ).

\subsubsection{Cross-modal matching}

In the cross-modal matching, the mean percentage of correct responses was $58.1 \%(\mathrm{SD}=26.8)$ for children with ASD and $80.1 \%(S D=21.4)$ for TD children. There was a significant difference between the ASD and TD groups (Mann-Whitney test: $Z=2.36, p=0.018, r=0.42)$.

\subsection{Differences between matching tasks in each group}

The percentage of correct responses in intramodal matching was higher than in cross-modal matching for both children with ASD (Wilcoxon signed-rank test: $Z=-2.25, p=0.024, r=0.71$ ) and TD children (Wilcoxon signed-rank test: $Z=-3.18$, $p=0.001, r=0.68)$.

\subsection{Group differences for four emotions}

\subsubsection{Intramodal matching}

In the intramodal matching, the children with ASD had the following mean percentages of correct responses: $82.5 \%$ (SD = 31.3) for "happy," 87.5\% (SD = 31.7) for "angry," 87.5\% (SD = 27.0) for "sad," and 82.5\% (SD = 31.3) for "surprised." The percentages for the TD children were as follows: $98.9 \%$ (SD = 3.1) for "happy," $100.0 \%$ (SD = 0.0) for "angry," $97.7 \%$ (SD = 7.4) for "sad," and 98.9\% (SD = 5.3) for "surprised" (see Table 1). There was no significant difference between the two groups with Bonferroni correction (happy: $Z=2.05, p=0.040, r=0.36$; angry: $Z=2.13, p=0.033, r=0.38$; sad: $Z=0.91, p=0.322, r=0.18$; surprised: $Z=2.05, p=0.040, r=0.36)$.

\subsubsection{Cross-modal matching}

In the cross-modal matching, the children with ASD had the following mean percentages of correct responses: $52.5 \%$ (SD = 46.3) for "happy," 55.0\% (SD = 35.0) for "angry," 85.0\% (SD = 26.9) for "sad," and 40.0\% (SD = 37.6) for "surprised." The percentages for the TD children were as follows: 75.0\% (SD = 34.5) for "happy," 79.5\% (SD = 31.5) for "angry," 97.7\% (SD = 7.4) for "sad," and 68.2\% (SD = 37.1) for "surprised" (see Table 1). There was no significant difference between the two groups with Bonferroni correction (happy: $Z=1.27, p=0.205, r=0.22$; angry: $Z=2.10, p=0.036, r=0.37 ;$ sad: $Z=1.61, p=0.108$, $r=0.28$; surprised: $Z=1.81, p=0.070, r=0.32$ ).

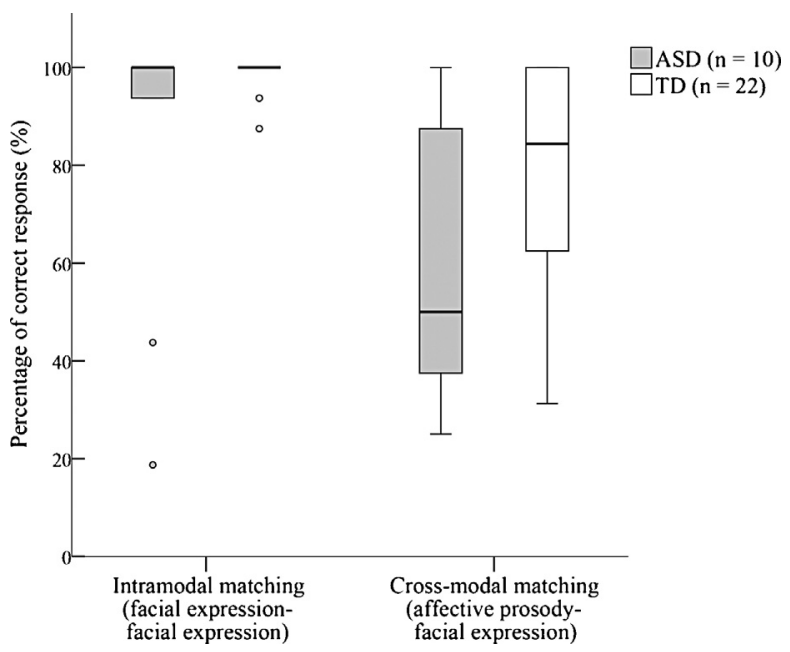

Fig. 1. Box plot display for the percentages of correct responses in each matching task. The lines in the box indicate the median of the distribution. The upper and lower boundaries of the boxes indicate the 25th and 75th percentiles, respectively, and the upper and lower whiskers indicate highest and smallest, respectively. 
Table 1

Confusion matrix for each of the four emotions in each group with responses in percentages for all sample emotions. Percentages of correct responses are underlined.

\begin{tabular}{|c|c|c|c|c|c|c|c|c|c|}
\hline \multirow[t]{3}{*}{ Group } & \multirow[t]{3}{*}{ Response } & \multicolumn{4}{|c|}{$\begin{array}{l}\text { Intramodal matching (facial expression-facial } \\
\text { expression) }\end{array}$} & \multicolumn{4}{|c|}{$\begin{array}{l}\text { Cross-modal matching (affective prosody-facial } \\
\text { expression) }\end{array}$} \\
\hline & & \multicolumn{4}{|l|}{ Sample } & \multicolumn{4}{|l|}{ Sample } \\
\hline & & Happy & Surprised & Angry & Sad & Happy & Surprised & Angry & Sad \\
\hline \multirow[t]{4}{*}{ ASD } & Happy & 82.5 & 2.5 & 7.5 & 2.5 & 52.5 & 25.0 & 17.5 & 5.0 \\
\hline & Surprised & $\overline{5.0}$ & 82.5 & 0.0 & 7.5 & $\overline{32.5}$ & 40.0 & 22.5 & 2.5 \\
\hline & Angry & 7.5 & 5.0 & 87.5 & 2.5 & 7.5 & 15.0 & 55.0 & 7.5 \\
\hline & Sad & 5.0 & 7.5 & $\overline{5.0}$ & $\underline{87.5}$ & 7.5 & 17.5 & $\overline{5.0}$ & $\underline{85.0}$ \\
\hline \multirow[t]{4}{*}{ TD } & Happy & 98.9 & 0.0 & 0.0 & 1.1 & 75.0 & 12.5 & 9.1 & 1.1 \\
\hline & Surprised & 1.1 & 98.9 & 0.0 & 1.1 & $\overline{10.2}$ & 68.2 & 6.8 & 1.1 \\
\hline & Angry & 0.0 & $\overline{0.0}$ & 100.0 & 0.0 & 5.7 & $\overline{9.1}$ & 79.5 & 0.0 \\
\hline & Sad & 0.0 & 1.1 & 0.0 & 97.7 & 9.1 & 10.2 & 4.5 & 97.7 \\
\hline
\end{tabular}

\subsection{Differences among the four emotions in each group}

\subsubsection{Intramodal matching}

Wilcoxon signed-rank tests showed no difference among the four emotions in intramodal matching for both children with ASD and TD children.

\subsubsection{Cross-modal matching}

For the children with ASD, Wilcoxon signed-rank tests showed a trend that the percentage of correct responses for sad was higher than that for the other three emotions; however, there was no significant difference with Bonferroni correction (happy vs. sad: $Z=-1.79, p=0.073, r=0.57$; surprised vs. sad: $Z=-2.38, p=0.017, r=0.75 ;$ angry vs. sad: $Z=-2.41, p=0.016$, $r=0.76$ ).

For the TD children, Wilcoxon signed-rank tests showed significant differences for happy vs. sad $(Z=-2.69, p=0.007$, $r=0.57)$ and surprised vs. sad $(Z=-3.10, p=0.002, r=0.66)$. There was also a trend toward the higher percentage of correct responses for sad than angry, but the difference did not remain significant with Bonferroni correction $(Z=-2.40, p=0.016$, $r=0.51)$.

\section{Discussion}

This study investigated emotion comprehension in young children with ASD and TD through the use of intramodal (visual stimulus-visual stimulus) and cross-modal (auditory stimulus-visual stimulus) matching. We used pictures of facial expressions as visual stimuli and spoken affective prosodies as auditory stimuli. The results revealed that both groups showed a high percentage of correct responses in intramodal matching. On the other hand, in cross-modal matching, children with ASD showed a lower percentage of correct responses than TD children.

In previous studies, adolescents with ASD exhibited high accuracy in both intramodal and cross-modal matching (Jones et al., 2011; Loveland et al., 1997). However, the results of this study revealed that young children with ASD, unlike adolescents, showed a lower accuracy in cross-modal matching than intramodal matching. The results of cross-modal matching also showed a difference in performance between children with ASD and TD children.

The participants in this study were younger (mean age: 6 years, 2 months) and had lower DAs (mean age: 5 years, 4 months) than in previous studies. The results of the present study showed the difficulty in learning the cross-modal relationship between affective prosody and facial expression at the early developmental stage. To promote emotion comprehension at this early developmental stage, cross-modal matching-to-sample training is necessary (Matsuda \& Yamamoto, 2013).

The intensity of facial expression may affect performance in intramodal matching. Previous studies indicated that individuals with ASD have deficits in understanding specific facial expressions with lower cue intensity (e.g., Evers, Noens, Steyaert, \& Wagemans, 2011; Humphreys, Minshew, Leonard, \& Behrmann, 2007; Law Smith, Montagne, Perrett, Gill, \& Gallagher, 2010; Rump, Giovannelli, Minshew, \& Strauss, 2009). In the study present here, children with ASD showed high percentages of correct responses for all four emotions in intramodal matching. This suggests that the intensities of facial expressions in this study were sufficient for children with ASD to understand.

Moreover, cue intensity of affective prosody may also affect the performance in cross-modal matching. Both children with ASD and TD children showed a higher percentage of correct response in sad than the other three emotions in cross-modal matching. This tendency was likely influenced by the cue intensity of affective prosody because the mean rating of sad affective prosody was higher than the others (Appendix). Future research is warranted to confirm how the intensity of affective prosody affects cross-modal matching in children with ASD. 
In conclusion, our results indicate a difference in the development of emotion comprehension between young children with ASD and TD children using intramodal and cross-modal matching. Children with ASD have difficulty understanding the relationship between auditory (affective prosody) and visual (facial expression) stimuli. On the other hand, they have no difficulty understanding the relationships between visual and visual stimuli, even when the faces of other persons are used. Based on these findings, the modality of the stimuli and responses, and the ages of children may have large effects on emotion comprehension in early developmental stage.

\section{Acknowledgements}

This research was supported by CREST research project on Social Imaging, JST. Soichiro Matsuda was supported by a fellowship from the Japan Society for the Promotion of Science.

\section{Appendix}

Mean ratings of emotions, pleasure, and arousal for stimuli based on 7-point scale and the Affect Grid (9-point scale). Ratings of predicted emotion are underlined.

\begin{tabular}{|c|c|c|c|c|c|c|c|}
\hline & & \multicolumn{3}{|c|}{ 7-point Likert scale } & \multicolumn{3}{|c|}{ The Affect Grid (9-point) } \\
\hline & & Happy & Surprised & Angry & Sad & Pleasure & Arousal \\
\hline \multirow{4}{*}{ Facial expression A } & Happy & 5.6 & 1.1 & 1.2 & 1.2 & 6.9 & 4.3 \\
\hline & Surprised & $\overline{1.2}$ & 6.6 & 2.7 & 2.6 & 2.6 & 7.6 \\
\hline & Angry & 1.1 & $\overline{1.7}$ & 6.6 & 1.8 & 0.8 & 7.3 \\
\hline & Sad & 1.2 & 1.5 & $\overline{1.4}$ & $\underline{6.4}$ & 1.4 & 4.1 \\
\hline \multirow[t]{4}{*}{ Facial expression B } & Happy & 5.6 & 1.9 & 1.4 & 1.5 & 6.3 & 5.2 \\
\hline & Surprised & $\overline{2.1}$ & 4.8 & 1.2 & 1.6 & 3.9 & 5.8 \\
\hline & Angry & 1.1 & $\overline{2.3}$ & 6.6 & 1.8 & 0.7 & 7.2 \\
\hline & Sad & 1.0 & 1.3 & $\overline{1.6}$ & $\underline{6.4}$ & 1.0 & 2.3 \\
\hline \multirow[t]{4}{*}{ Affective prosody } & Happy & 4.6 & 2.4 & 1.0 & 2.4 & 5.6 & 4.6 \\
\hline & Surprised & $\overline{1.3}$ & $\underline{5.5}$ & 2.1 & 2.0 & 3.0 & 7.2 \\
\hline & Angry & 1.9 & $\overline{1.9}$ & 4.2 & 2.6 & 2.5 & 5.1 \\
\hline & Sad & 1.1 & 1.6 & $\overline{1.4}$ & 6.7 & 0.6 & 2.3 \\
\hline
\end{tabular}

\section{References}

American Psychiatric Association (2000). Diagnostic and statistical manual of mental disorders (4th ed., Text Revised). Arlington, VA: Author.

American Psychiatric Association (2013). Diagnostic and statistical manual of mental disorders (5th ed.). Arlington, VA: Author.

Baker, K. F., Montogomery, A. A., \& Abramson, R. (2010). Brief report: Perception and lateralization of spoken emotion by youths with high-functioning forms of autism. Journal of Autism and Developmental Disorders, 40, 123-129.

Bormann-Kischkel, C., Vilsmeier, M., \& Baude, B. (1995). The development of emotional concepts in autism. Journal of Child Psychology and Psychiatry, 36, 12431259.

Boucher, J., Lewis, V., \& Collis, G. M. (2000). Voice processing abilities in children with autism, children with specific language impairments, and young typically developing children. Journal of Child Psychology and Psychiatry, 41, 847-857.

Braverman, A., Fein, D., Lucci, D., \& Waterhouse, L. (1989). Affect comprehension in children with pervasive developmental disorders. Journal of Autism and Developmental Disorders, 19, 301-316.

Capps, L., Yirmiya, N., \& Sigman, M. (1992). Understanding of simple and complex emotions in non-retarded children with autism. Journal of Child Psychology and Psychiatry, 33, 1169-1182.

Castelli, F. (2005). Understanding emotions from standardized facial expressions in autism and normal development. Autism, 9, 428-449.

Celani, G., Battacchi, M. W., \& Arcidiacono, L. (1999). The understanding of the emotional meaning of facial expressions in people with autism. Journal of Autism and Developmental Disorders, 29, 57-66.

Davies, S., Bishop, D., Manstead, A. S. R., \& Tantam, D. (1994). Face perception in children with autism and Asperger's syndrome. Journal of Child Psychology and Psychiatry, 35, 1033-1057.

Ekman, P. (1992). An argument for basic emotions. Cognition and Emotion, 6, 169-200.

Ekman, P., Friesen, W. V., O’Sullivan, M., Chan, A., Diacoyanni-Tarlatzis, I., Heider, K., et al. (1987). Universals and cultural differences in the judgments of facial expressions of emotion. Journal of Personality and Social Psychology, 53, 712-717.

Evers, K., Noens, I., Steyaert, J., \& Wagemans, J. (2011). Combining strengths and weaknesses in visual perception of children with an autism spectrum disorder: Perceptual matching of facial expressions. Research in Autism Spectrum Disorders, 5, 1327-1342.

Fink, E., de Rosnay, M., Wierda, M., Koot, H. M., \& Begger, S. (2014). Brief report: Accuracy and response time for the recognition of facial emotions in a large sample of children with autism spectrum disorders. Journal of Autism and Developmental Disorders, 44, 2363-2368.

Fritz, C. O., Morris, P. E., \& Richler, J. J. (2012). Effect size estimates: Current use, calculations, and interpretation. Journal of Experimental Psychology: General, 141, $2-18$.

Gepner, B., Deruelle, C., \& Grynfeltt, S. (2001). Motion and emotion: A novel approach to the study of face processing by young autistic children. Journal of Autism and Developmental Disorders, 31, 37-45.

Grossman, J. B., Klin, A., Carter, A. S., \& Volkmar, F. R. (2000). Verbal bias in recognition of facial emotions in children with Asperger syndrome. Journal of Child Psychology and Psychiatry, 41, 369-379.

Grossman, R. B., Bemis, R. H., Plesa Skwerer, D., \& Tager-Flusberg, H. (2010). Lexical and affective prosody in children with high-functioning autism. Journal of Speech, Language, and Hearing Research, 53, 778-793.

Hobson, R. P. (1986a). The autistic child's appraisal of expressions of emotion. Journal of Child Psychology and Psychiatry, $27,321-342$.

Hobson, R. P. (1986b). The autistic child's appraisal of expressions of emotion: A further study. Journal of Child Psychology and Psychiatry, 27, 671-680. 
Humphreys, K., Minshew, N., Leonard, G. L., \& Behrmann, M. (2007). A fine-grained analysis of facial expression processing in high functioning adults with autism. Neuropsychologia, 45, 685-695.

Ikuzawa, M., Matsushita, Y., \& Nakase, A. (Eds.). (2002). Kyoto scale of psychological development 2001. Kyoto: Kyoto International Social Welfare Exchange Centre (in Japanese).

Jones, C. R. G., Pickles, A., Falcaro, M., Marsden, A. J. S., Happé, F., Scott, S. K., et al. (2011). A multimodal approach to emotion recognition ability in autism spectrum disorders. Journal of Child Psychology and Psychiatry, 52, 275-285.

Koyama, T., Osada, H., Tsujii, H., \& Kurita, H. (2009). Utility of the kyoto scale of psychological development in cognitive assessment of children with pervasive developmental disorders. Psychiatry and Clinical Neurosciences, 63, 241-243.

Lacroix, A., Guidetti, M., Rogé, B., \& Reilly, J. (2009). Recognition of emotional and nonemotional facial expressions: A comparison between Williams syndrome and autism. Research in Developmental Disabilities, 30, 976-985.

Law Smith, M. J., Montagne, B., Perrett, D. I., Gill, M., \& Gallagher, L. (2010). Detecting subtle facial emotion recognition deficits in high-functioning autism using dynamic stimuli of varying intensities. Neuropsychologia, 48, 2777-2781.

Loveland, K. A., Tunali-Kotoski, B., Chen, Y. R., Ortegon, J., Pearson, D. A., Brelsford, K. A., et al. (1997). Emotion recognition in autism: Verbal and nonverbal information. Development and Psychopathology, 9, 579-593.

Macdonald, H., Rutter, M., Howlin, P., Rios, P., Le Conteur, A., Evered, C., et al. (1989). Recognition and expression of emotional cues by autistic and normal adults. Journal of Child Psychology and Psychiatry, 30, 865-877.

Matsuda, S., \& Yamamoto, J. (2013). Intervention for increasing the comprehension of affective prosody in children with autism spectrum disorders. Research in Autism Spectrum Disorders, 7, 938-946.

Matsuda, S., \& Yamamoto, J. (2014). Computer-based intervention for inferring facial expressions from socio-emotional context in two children with autism spectrum disorders. Research in Autism Spectrum Disorders, 8, 944-950.

Paul, R., Augustyn, A., Klin, A., \& Volkmar, F. R. (2005). Perception and production of prosody by speakers with autism spectrum disorders. Journal of Autism and Developmental Disorders, 35, 205-220.

Peppé, S., McCann, J., Gibbon, F., O'Hare, A., \& Rutherford, M. (2007). Receptive and expressive prosodic ability in children with high-functioning autism. Journal of Speech, Language, and Hearing Research, 50, 1015-1028.

Prior, M., Dahlstrom, B., \& Squires, T. (1990). Autistic children's knowledge of thinking and feeling states in other people. Journal of Child Psychology and Psychiatry, 31, 587-601.

Rosset, D. B., Rondan, C., Da Fonseca, D., Santos, A., Assouline, B., \& Deruelle, C. (2008). Typical emotion processing for cartoon but not for real faces in children with autistic spectrum disorders. Journal of Autism and Developmental Disorders, 38, 919-925.

Rump, K., Giovannelli, J. L., Minshew, N. J., \& Strauss, M. S. (2009). The development of emotion recognition in individuals with autism. Child Development, 80, $1434-1447$.

Russell, J. A., Weiss, A., \& Mendelsohn, G. A. (1989). Affect grid: A single-item scale of pleasure and arousal. Journal of Personality and Social Psychology, 57, $493-502$.

Tanaka Institute for Educational Research (2003). Tanaka Binet Chinou Kensa V. [Tanaka-Binet intelligence scale V]. Tokyo: Taken Publishing (in Japanese).

Tantam, D., Monaghan, L., Nicholson, H., \& Stirling, J. (1989). Autistic children's ability to interpret faces: A research note. Journal of Child Psychology and Psychiatry, $30,623-630$ 